\title{
Theoretical Minimum Detection Range for a Rapidly Moving Target and an Experimental Evaluation
}

\author{
Ilkyu Kim ${ }^{1} \cdot$ Hyun Kim² $\cdot$ Jeong-Hae Lee,
}

\begin{abstract}
There have been considerable challenges with radar systems for detecting high-speed projectiles at a short range. This necessitates the presentation of a guideline for the minimum detection range that will guarantee detection of a target. In this letter, the detection range for a rapidly flying target is studied based on the characteristics of the target, such as speed and launch angle, and radar parameters such as pulse repetition interval, dwell time, beamwidth, and so on. The derived equation was applied to parametric studies for different characteristics of targets in order to investigate the influential parameters that affect the minimum detection range. A field test using the radar system's prototype was performed to evaluate the validity of the proposed equation.
\end{abstract}

Key Words: Minimum Detection Range, Radar System, Rapid Target.

\section{INTRODUCTION}

One of the important functions of a radar system is the detection of a fast-moving target launched from the ground. The probability of detecting a target is mostly determined by parameters such as the frequency band, antenna type, and waveforms. A radar system is capable of sequentially allocating a pencilshaped beam to sectors along a horizontal line. When a launched object passes over the sectors, the radar system can detect it, track its path, and provide queuing information (position, direction, speed, etc.). A radar system needs to recognize the presence of a rapidly moving target before it quickly passes through the search sector. For such an application, the minimum detection range is a function of various parameters such as target speed and launch angle, including the pulse repetition interval (PRI), beamwidth, and dwell time. However, previous studies have focused on the calculation of the minimum range based on the blindness of the receiver, which is determined by the transmit pulse width [1]. Alert-confirm detection has been investigated with respect to optimum dwell time and reduced false alarm probabilities [2]. However, to the best of our knowledge, no comprehensive research has been conducted that focused on the range of detection and took into account the characteristics of the target.

In this letter, the minimum detection range of a radar system is studied based on the geometry of the target. A radar system often fails to detect a rapid object at a short range that is greater than the one calculated by the blindness of the receiver. This work deals with a projectile that can move at a speed of hundreds of meters per second. In order to avoid missing such a target, it is necessary to provide guidelines on the minimum detection ranges for rapidly moving targets. This letter discusses

Manuscript received March 17, 2020 ; Revised December 29, 2020 ; Accepted March 12, 2021. (ID No. 2020317-033J)

${ }^{1} \mathrm{C} 4 \mathrm{I}$ Team, Defense Agency for Technology and Quality, Jinju, Korea.

${ }^{2}$ Radar R\&D, LIG Nex1 Co., Yongin, Korea.

${ }^{3}$ Department of Electronics and Electrical Engineering, Hongik University, Seoul, Korea.

"Corresponding Author: Jeong-Hae Lee (e-mail: Jeonglee@hongik.ac.kr)

This is an Open-Access article distributed under the terms of the Creative Commons Attribution Non-Commercial License (http://creativecommons.org/licenses/by-nc/4.0) which permits unrestricted non-commercial use, distribution, and reproduction in any medium, provided the original work is properly cited.

(c) Copyright The Korean Institute of Electromagnetic Engineering and Science. All Rights Reserved. 
theoretical derivations and parametric studies on the minimum detection range, as well as the results of field tests that were conducted to validate the calculations.

\section{DERIVATION OF MINIMUM DETECTION RANGE}

The minimum detection range can be derived as an aspect of the pulse width of the transmitter and the geometries of the target detection. First, a receiver is restricted to listening to returning echoes during the pulse width of the transmitter. This blindness of the receiver leads to a minimum detection range of

$$
R_{\min }=\frac{\tau_{t} c}{2}
$$

where $\tau_{t}$ is the pulse width of the transmitter, and $c$ is the speed of light. Second, the detection range is derived from the geometry of the detection as described in Fig. 1.

In order to meet the requirements for the probability of detection, an adequate number of PRI beams must be in use when the target passes through the sectors along a horizontal line. Thus, the time duration $T_{\text {pass }}$ can be defined as

$$
T_{\text {pass }} \geq N_{P R I} \times T_{\text {scan }}
$$

The scanning time duration $T_{\text {scan }}$ can be written as

$$
T_{\text {scan }}=N_{\text {sector }} \times T_{\text {dwell }}
$$

where $N_{\text {sector }}$ is the number of sectors covered by the radar above the horizontal line, and $T_{\text {dweel }}$ is the length of time that a pencil beam dwells in each sector. The duration that $T_{\text {pass }}$ will be passing through the beam cross-section can then be calculated based on the geometries depicted in Fig. 1 by using Eq. (4).

$$
T_{\text {pass }}=\frac{R \times \sin \left(\theta_{e l} / 2\right)}{M_{v} \times \sin \theta_{t}}\left(\frac{1}{\sin \left(\theta_{t}+\theta^{\prime}+\frac{\theta_{e l}}{2}\right)}+\frac{1}{\sin \left(\theta_{t}+\theta^{\prime}\right)}\right)
$$

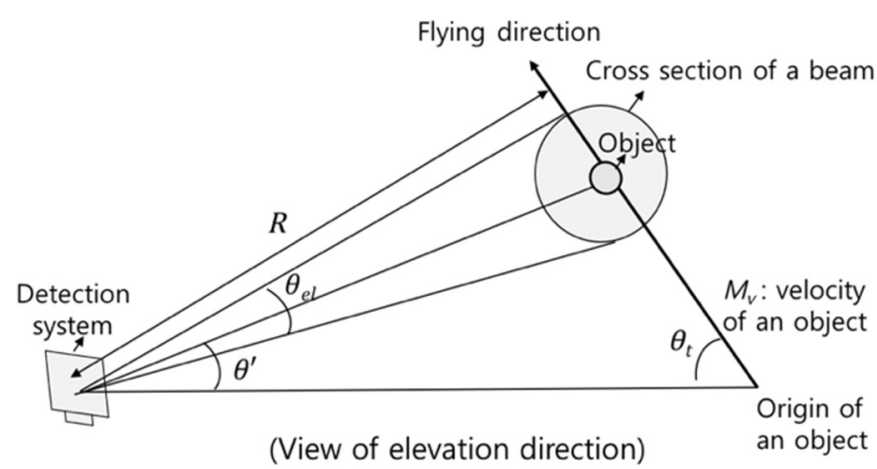

Fig. 1. Geometries of a rapidly moving object. where $M_{v}$ and $\theta_{t}$ are the speed and launch angle for the object, respectively, and $R$ and $\theta_{e l}$ are the detection range from the radar system to the object and the $3 \mathrm{~dB}$ beamwidth for the radar system, respectively. The angle $\theta^{\prime}$ is the elevation angle between the ground and the target. For surveillance radar systems, the detection is made along a horizontal line, which assumes that the angle $\theta^{\prime}$ is zero. The parametric studies discussed in Section III were performed based on the assumption that substituting Eqs. (3) and (4) into Eq. (2) will produce the detection range $R$ (Table 1 ). The minimum detection range $R_{\min }$ can be defined as the smallest detection range $R$ by using the following equation.

$$
\begin{aligned}
R_{\min }= & \frac{M_{v}}{\sin \left(\theta_{e l} / 2\right)}\left(\frac{1}{\sin \left(\theta_{t}+\theta^{\prime}+\frac{\theta_{e l}}{2}\right)}+\frac{1}{\sin \left(\theta_{t}+\theta^{\prime}\right)}\right)^{-1} \\
& \times N_{P R I} \times N_{\text {sector }} \times T_{\text {dwell }}
\end{aligned}
$$

The derivation in Eq. (5) is based on the assumption that the signal-to-noise ratio (SNR) is satisfied because the detection range of interest is much closer than the maximum one. The minimum detection range in Eq. (5) can be used when the range is greater than the blind range in Eq. (1).

\section{PARAMETRIC STUDIES ON THE DETECTION RANGE}

It is desirable to reduce the minimum detection range in terms of the operational aspects of a target detection system. In principle, the minimum detection ranges can be defined based on Eq. (1), as with the representative examples provided in the following:

i) $\mathrm{PRI}=350 \mu \mathrm{s}$, Tx pulse width $=35 \mu \mathrm{s}$, and $R_{\text {min }}=5.25 \mathrm{~km}$.

ii) $\mathrm{PRI}=200 \mu \mathrm{s}$, Tx pulse width $=20 \mu \mathrm{s}$, and $R_{\min }=3 \mathrm{~km}$.

When the above ranges are smaller than the ones obtained from the geometries of the rapidly moving object, the radar system is likely to miss the target at the above range. The geometry

Table 1. Typical parameters of detection systems and objects

\begin{tabular}{lc}
\hline Parameter & Value \\
\hline$M_{v}(\mathrm{~m} / \mathrm{s})$ & $300-800$ \\
$\theta_{t}(\mathrm{deg})$ & $30-60$ \\
$T_{\text {duell }}(\mathrm{ms})$ & $7.1-9.1$ \\
$\theta_{l}(\mathrm{deg})$ & $1-7$ \\
$N_{P R I}$ & $3-5$ \\
$N_{\text {settor }}$ & $25-40$ \\
\hline
\end{tabular}




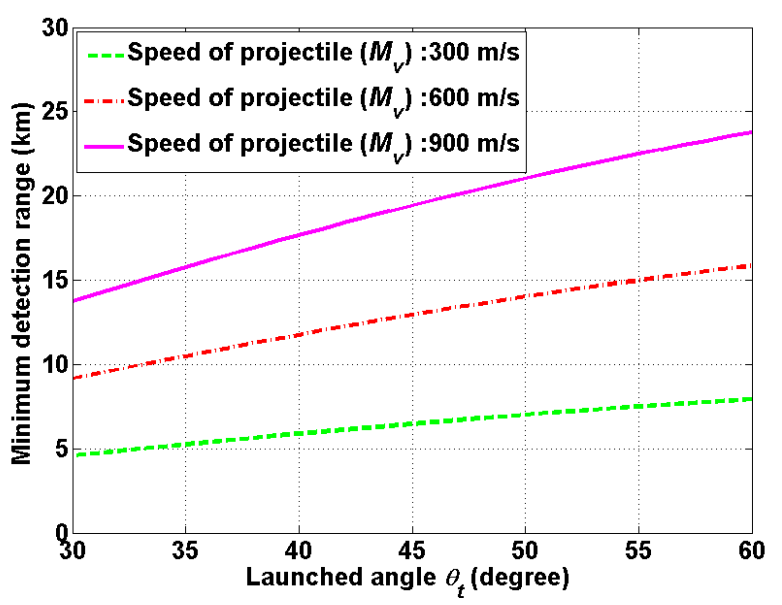

Fig. 2. Minimum detection range in terms of $M_{v}$ and $\theta_{t}$.

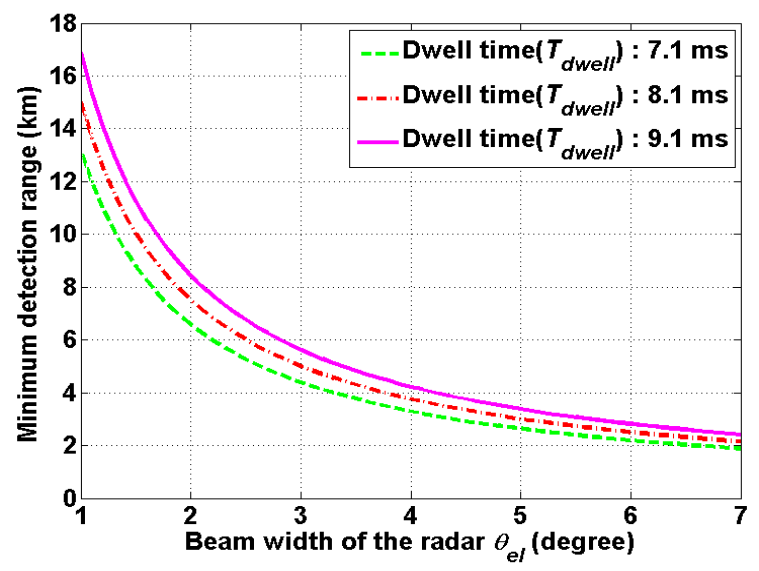

Fig. 3. Minimum detection range in terms of $T_{\text {dwell }}$ and $\theta_{\text {el. }}$

of the target can be emphasized more for a system in which it is required to detect a projectile moving at high speeds. The first case of the study was the calculation of the minimum range according to the launched angle and the speed of the projectile. As illustrated in Fig. 2, when the launch angle was increased, the minimum range also increased due to a reduction of $T_{\text {pass }}$ for each beam sector. In addition, it was observed that a faster target led to a higher minimum detection range. The parameter settings $T_{\text {dwell }}=7.1 \mathrm{~ms}, \theta_{c l}=2.0^{\circ}$, $N_{\text {sector }}=30$, and $N_{P R I}=5$ were used for this case study. The second case involved the settings of the beamwidth and dwell time. As shown in Fig. 3, the exploitation of a greater beamwidth allowed the $T_{\text {pass }}$ in each beam sector to be increased. It was observed that an increase in the duration $T_{\text {pass }}$ reduced the minimum detection range. In contrast, the use of a longer dwell time increased an entire duration of the search with all beam sectors. The longer dwell time led to the increase in the minimum detection range. Note that the parameter settings $\theta_{t}=46^{\circ}, M_{v}=300 \mathrm{~m} / \mathrm{s}, N_{\text {sector }}=30$, and $N_{P R I}=5$ were used in this evaluation.

The third case was the calculation of the minimum detection range in terms of the number of sectors and PRI beams.
Fig. 4 depicts the variation of the minimum detection range with respect to the number of sectors and PRI beams. The minimum detection range increased as the number of sectors and/or PRI beams increased. This is because more assigned sectors and PRI beams led to an increase in the search duration. The parameter settings $T_{\text {dwell }}=7.1 \mathrm{~ms}, \theta_{e l}=2.0^{\circ}, \theta_{t}=$ $46^{\circ}$, and $M_{v}=300 \mathrm{~m} / \mathrm{s}$ were used in the last case study.

The field test was performed using the prototype of a radar system. Fig. 5 shows the trajectory of a projectile obtained by the field test. The radar system tracked a flying projectile until the detection range was as short as possible. The distance between the radar system and the origin of the object was set as $5 \mathrm{~km}$, and the validity of the calculated minimum range $R_{\text {cal }}$ was verified by comparing it to the minimum range $R_{\text {mea }}$ measured in the field test. The minimum detection range obtained from the trajectory in Fig. 5 is the first case provided in Table 2, which reveals that both cases showed agreement at less than $5 \%$ deviation. This radar system was designed to search and track above the horizontal line to reduce adverse effects from geometric clutter. The short-range detection is less affected by time variances in atmospheric reflectivity and any estimation error.

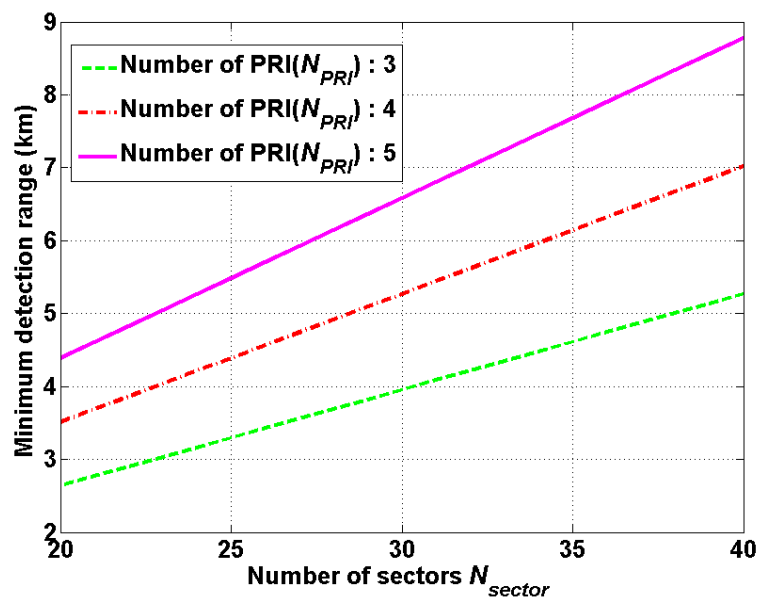

Fig. 4. Minimum detection range in terms of $N_{\text {sector }}$ and $N_{P R I}$.

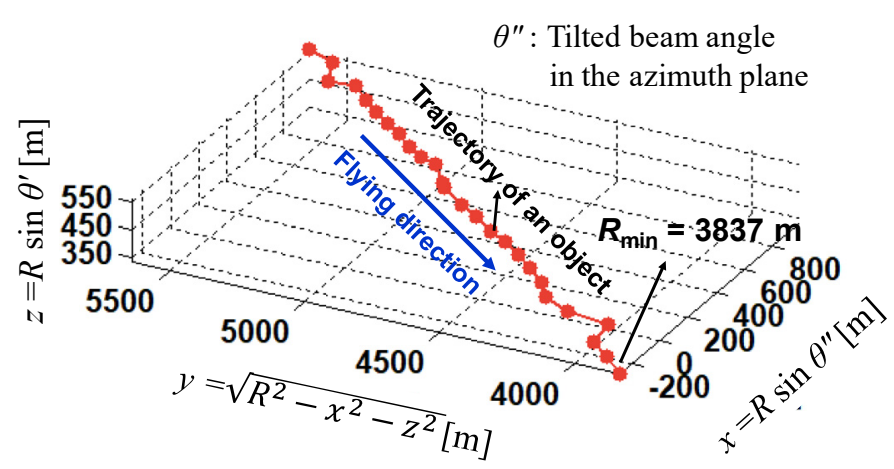

Fig. 5. The projectile trajectory as measured with a field test. 
Table 2. Comparison of calculated and measured results

\begin{tabular}{lcc}
\hline & $R_{\text {cal }}(\mathrm{km})$ & $R_{\text {mea }}(\mathrm{km})$ \\
\hline Case $1\left(M_{v}=297 \mathrm{~m} / \mathrm{s}, \theta_{t}=18^{\circ}, N_{P R I}=4\right)$ & 3.62 & 3.8 \\
Case $2\left(M_{v}=350 \mathrm{~m} / \mathrm{s}, \theta_{t}=23^{\circ}, N_{P R I}=4\right)$ & 5.35 & 5.4 \\
\hline
\end{tabular}

\section{CONCLUSION}

In this letter, a simple equation for the minimum detection range of a rapidly moving target was derived based on the geometry of the rapid movement. The use of this formula means that parametric studies could be performed to determine the minimum detection range, and our field test results validated the effectiveness of the equation.

\section{Ilkyu Kim}

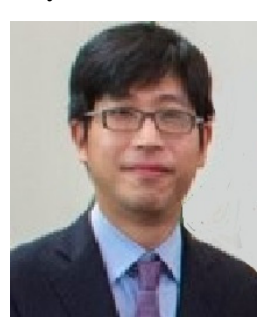

received his B.S. degree in electrical engineering from Hongik University, Seoul, South Korea in 2003 and the M.S. degree in electrical engineering from University of Southern California, Los Angeles, CA in 2006 and Ph.D. degrees in electrical engineering from University of California at Los Angeles in 2012. He was with Samsung Advanced Institute of Technology from 2006 to 2008. After gaining his Ph.D. degree, he joined in Defense Agency for Technology Quality where he is currently working as a team leader of $\mathrm{C} 4 \mathrm{I}$ technology planning team. His research interests include but not limited to computation of electromagnetic mutual coupling, antenna measurement, and antenna design for space and radar applications.

\section{Hyun Kim}

Photograph and biography not available at the time of publication.
The research work of the author Jeong-Hae Lee was supported by Basic Science Research Program through Ministry of Education (No. 2015R1A6A1A03031833).

\section{REFERENCES}

[1] S. H. Al Sadoon and B. H. "Radar theoretical study: minimum detection range and maximum signal to noise ratio (SNR) equation by using MATLAB simulation program, " American Journal of Modern Physics, vol. 2, no. 4, pp. 234241, 2013.

[2] E. H. Kim and J. Park, "Dwell time optimization of alertconfirm detection for active phased array radars," Journal of Electromagnetic Engineering and Science, vol. 19, no. 2, pp. 107-114, 2019.

Jeong-Hae Lee

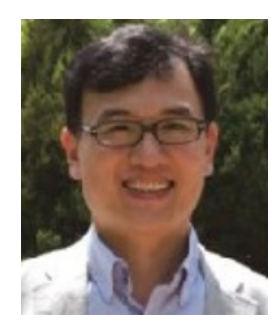

received B.S. and M.S. degrees in electrical engineering from Seoul National University in Korea in 1985 and 1988, respectively, and a Ph.D. degree in electrical engineering from the University of California, LA in 1996. From 1993 to 1996, he was a visiting scientist of general atomics in San Diego, CA, where his major research initiatives were developing a millimeter-wave diagnostic system and studying plasma wave propagation. Since 1996, he has been working at Hongik University in Seoul, Korea as a professor in the Department of Electronic and Electrical Engineering. He has more than 100 papers published in journals and 60 patents. He was a president of the Korea Institute of Electromagnetic Engineering and Science in 2019. He is currently a director of the Metamaterial Electronic Device Center. His current research interests include metamaterial radio frequency devices and wireless power transfer. 